\title{
The Professional Quality of Life of Nurses who Work in the Intensive Care Unit and Emergency Unit
}

\author{
Astri Eka Wulandari, F. Sri Susilaningsih, Irman Somantri \\ Faculty of Nursing, Universitas Padjadjaran \\ Email : astriekaw0@gmail.com
}

\begin{abstract}
Nurses need compassion when providing health services. Compassion felt by nurses can be Compassion Satisfaction (positive aspects) and Compassion Fatigue (negative aspects), and may affect nursing care and Nurses' Professional Quality of Life. The tendency to negative aspects will be felt by the nurses themselves, patients and hospitals. The purpose of this study was to determine the quality of life of professionals (Professional Quality of Life) nurses in the Intensive Care Unit (ICU) and Emergency Room (ER) of the Kota Bandung Hospital. This research was a quantitative descriptive study. Involving 62 nurses from ICU and ER through total sampling technique with a response rate of 97\%. Data was collected using Professional Quality of Life (ProQol) version 5 then analyzed with descriptive statistics. The results showed that $20.97 \%$ had a positive Professional Quality of Life according to the Stamm combination interpretation while outside of Stamm's interpretation, $32.26 \%$ tended to be positive. According to the results of the average score of each sub-variable, Compassion Satisfaction was in the medium category. The highest average score of compassion satisfaction was ICU nurses, the highest secondary traumatic stress was PICU/NICU nurses and the highest average score of burnout was emergency nurses. Hospitals need to conduct regular reviews of nurse Professional Quality of Life and conduct counseling programs for nurses who have ProQol towards negative and risky aspects.
\end{abstract}

Keywords: Emergency room, intensive care unit, nurses, professional quality of life. 
Astri Eka: The Professional Quality of Life of Nurses who Work in the Intensive Care Unit

\section{Introduction}

Nurses are professional health workers who play an important role in meeting the basic needs of patients. Professional nurses are required to be caring to patients, which is indicated by being there when patients are needed, adjusting to the patient's condition, and being directly involved in patient care (Schroeder, 2011). The presence of nurses for example by listening to patients about their pain, fear, or suffering that is being experienced by patients.

Nursing care should an optimal service so that clients' needs are met, and as a result, clients would be independent. The optimum nursing care provided gives a feeling of satisfaction for nurses. Satisfaction felt by nurses related to their profession is called Professional Quality of Life (ProQOL). When nurses have a negative professional quality of life that would be impacted patients' health (Stamm, 2010), for example, nursing care provided by nurses is not optimal.

The professional quality of life includes positive and negative aspects that are influenced by the work environment, clients, and individuals (Stamm, 2010). Positive aspects are illustrated by the satisfaction felt by nurses when providing the best service called compassion satisfaction. Nurses who feel or have a high value of compassion satisfaction are a reflection of their involvement in their work and good quality of professional life.

Compassion satisfaction is positively related to the ability to communicate (good communication among nurses, colleagues or patients), collaboration (contributing fully and making the work environment more positive so that there is a link between team members) and having leadership where high compassion satisfaction from nurses is supported by welfare, appreciation, achievement, joy, gratitude, and hope (Kelly \& Todd, 2017; Sacco \& Copel, 2017). A challenge for nurses to achieve a good professional quality of life is a negative aspect that is felt by a nurse, namely compassion fatigue. Compassion fatigue is divided into two parts, Burnout and Secondary Traumatic Stress (Stamm, 2010).

Compassion fatigue is very vulnerable for nurses because nurses enter into other people's life (patients and families) deeply and become partners of patients through their illnesses. Moreover, nurses are responsible for treating patients who are sick, injured, traumatized, or vulnerable conditions. This exposes them to exposure to pain, trauma, and suffering routinely (Hooper, Craig, Janvrin, Wetsel, \& Reimels, 2010). Signs and symptoms of fatigue compassion are not recognized by nurses or co-workers (Crewe \& Crewe, 2016).

Compassion fatigue is recognized as a problem in emergency nurses, emergency departments, patients and families (Emergency Nurses Association, 2014). According to Ariapooran (2014), emergency nurses are more susceptible to experiencing or at risk of experiencing compassion fatigue compared to non-emergency nurses. Nurses in intensive care, nephrology and oncology are also at risk of feeling higher fatigue compassion than the value of compassion satisfaction (Hooper et al., 2010).

Berger et al. (2015) study showed that the average of burnout and secondary traumatic stress in emergency and intensive care nurses showed higher than compassion satisfaction. Sacco et al. (2015) study showed that the average critical care nurse has high compassion satisfaction accompanied by high burnout and secondary traumatic stress. The possibility of nurses experiencing fatigue compassion in the emergency and intensive units because these room manages a lot of patients in critical, life-threatening conditions, and needs immediate help. Nurses are more at risk with trauma or death. Nurses who work in the emergency department on average handle 50 patients per shift with various disease cases (Braunschneuider, 2013). In contrast to the conditions in the inpatient room, the room is focused more patients in the recovery phase.

The city of Bandung has a public hospital managed by the government, namely the Regional General Hospital (RSUD) of Bandung City as a level 2 health facility. From the hospital observation, nurses in the emergency unit in this hospital handle many patients with unpredictable and crowded conditions. While nurses in the intensive care unit should observe patients every second. Nurses have the potential to experience fatigue compassion, which would 
Astri Eka: The Professional Quality of Life of Nurses who Work in the Intensive Care Unit

affect professionals' quality of life. This study aimed to assess the quality of life of Professional Quality of Life in ICU and ER of Bandung City Hospital.

\section{Research Method}

The research was a descriptive quantitative study. The population of 64 nurses was divided into 15 nurses in the ICU, 21 nurses of the Neonatal Intensive Care Unit (NICU) and Pediatric Intensive Care Units (PICU) and 28 nurses in ER. The study was conducted on 62 nurses due to 1 unwilling to participate and 1 on leave. Therefore the overall response rate was $97 \%$. Data was taken using the Professional Quality of Life (ProQOL) version 5 instrument which is a standard instrument from Stamm (2010) and is translated into Indonesian by Debora et al. (2017). The instrument consists of 30 statements with a Likert scale 1 (never) to 5 (very often). Calculation of the results of the Stamm ProQol-5 questionnaire (2010) is divided into three ranges of values for the three sub-variables (compassion satisfaction, burnout, and secondary traumatic stress), namely the low category $(\leq 22)$, moderate (23$41)$, and height $(\geq 42)$. This research received ethical approval from the Research Ethics Commission of Padjadjaran University with an ethical license number 710/UN6.KEP/ $\mathrm{EC} / 2018$.

\section{Research Results}

Table 1 Characteristic of Respondents $(n=62)$

\begin{tabular}{lccc}
\hline \multicolumn{1}{c}{ Characteristic } & ER & ICU & PICU/NICU \\
\hline Gender & $\mathbf{n ~ ( \% )}$ & $\mathbf{n}(\mathbf{\%})$ & $\mathbf{n}(\mathbf{\%})$ \\
Male & $16(61.5)$ & & $4(19)$ \\
Female & $10(38.5)$ & $6(40)$ & $17(81)$ \\
Age & & $9(60)$ & $9(60)$ \\
$\leq 35$ & $22(84.6)$ & $10(47.6)$ & $6(40)$ \\
$>35$ & $4(15.4)$ & $11(52.4)$ & \\
\hline
\end{tabular}

Table 2 Nurses' Professional Quality of Life in ICU and ER $(n=62)$

\begin{tabular}{lcc}
\hline \multicolumn{1}{c}{ Professional Quality of Life } & $\begin{array}{c}\text { Frekuensi } \\
(\mathbf{f})\end{array}$ & $\begin{array}{c}\text { Persentase } \\
(\mathbf{\%})\end{array}$ \\
\hline The interpretation of ProQol-5 & & 20.97 \\
Stamm 2010 combination & 13 & 1.61 \\
Positive & 1 & 0 \\
High risk & 0 & 0 \\
Overwhelmed & 0 & 0 \\
Unique & 0 & \\
Distress & & 24.19 \\
The interpretation of ProQol-5 & & 32.26 \\
Stamm 2010 out of the & 15 & 20.97 \\
combination & 20 & \\
The balance of nurses' QoL & 13 & \\
Tend to be positive's perceptions & & \\
High risk of Compassion Fatigue & & \\
\hline
\end{tabular}


Astri Eka: The Professional Quality of Life of Nurses who Work in the Intensive Care Unit

Table 3 Nurses'Professional Quality of Life -based on Sub-variables (n=62)

\begin{tabular}{lll}
\hline \multicolumn{1}{c}{$\begin{array}{c}\text { Sub-variable Professional } \\
\text { Quality of Life }\end{array}$} & Mean \pm SD & criteria \\
\hline Compassion Satisfaction & $38.58 \pm 5.12$ & Moderate (23-41) to high $(\geq 42)$ \\
Burnout & $21.69 \pm 4.97$ & Low $(\leq 22)$ to moderate $(23-41)$ \\
Secondary traumatic stress & $20.61 \pm 3.99$ & Low $(\leq 22)$ to moderate $(23-41)$ \\
\hline
\end{tabular}

Table 4 Nurses' Professional Quality of Life-based on Rooms Criteria ( $\mathrm{n}=62$ )

\begin{tabular}{lccccccc}
\hline \multicolumn{1}{c}{ Rooms } & N & \multicolumn{2}{c}{ Compassion Satisfaction } & \multicolumn{2}{c}{ Burnout } & \multicolumn{2}{c}{ Secondary Traumatic Stress } \\
\hline & & Mean & SD & Mean & SD & Mean & SD \\
\hline ER & 26 & 36.42 & 5.27 & 23.58 & 5.21 & 19.88 & 4.55 \\
ICU & 15 & 40.27 & 5.29 & 19.93 & 4.80 & 20.53 & 3.72 \\
PICU/NICU & 21 & 40.05 & 3.90 & 20.62 & 4.14 & 21.57 & 3.37 \\
\hline
\end{tabular}

Table 1 shows the gender characteristics, there were more male nurses in the emergency room, and in terms of age, more IGD and PICU/NICU nurses were nurses 35 years old. Table 2 presents there were only 14 nurses who are included in the Professional Quality of Life Stamm combination, in contrast, the majority of respondents were interpreted in the outside combination of the Stamm.

Table 3 shows that the Professional Quality of Life from 62 nurses seen that the three sub-variables had an average score of Compassion Satisfaction higher than the average score of Burnout and Secondary Traumatic Stress. Table 4 describes that the sub-variable Compassion Satisfaction had the lowest average score in the emergency room and the highest in the ICU. The results in the three rooms were in the medium category. The lowest average score of Burnout (low category) was in the ICU and the highest (moderate category) in the emergency room. For the variable Secondary Traumatic Stress, the lowest average score was in the emergency room and the highest in the PICU/ NICU space even though all three were in a low category $(\leq 22)$.

\section{Discussion}

The results show that 14 nurses filled out questionnaires based on the Professional Quality of Life version 5. Stamm (2010) suggested that the Professional Quality of Life is positive when compassion satisfaction is high (score $\geq 42$ ), and nurses' burnout and secondary traumatic are low to moderate levels (20.97\%). Professional Quality of Life standards should match those set by Stamm in 2010. In this study, $77.42 \%$ of nurses still have moderate compassion satisfaction or 48 nurses cannot be categorized into the Stamm combination interpretation category. Therefore, this study found nurses who have moderate compassion satisfaction, so hospitals need to make efforts to make compassion satisfaction increase accompanied by low fatigue compassion which would make the Professional Quality of Life a positive nurse. Efforts can be made by hospitals such as regular education, counseling programs, improving nurse welfare, Certified Compassion Fatigue Specialist Training (CCFST) training. According to several studies, it was effective in improving compassion satisfaction.

The findings in this study showed that $32.26 \%$ of nurses have a positive professional quality of life because of moderate compassion satisfaction level and low fatigue compassion level. This study is in line with the results of Sacco et al. (2015), according to him, if burnout and secondary traumatic stress do not have high values, Professional Quality of Life can be positive. Austiny et al. (2017) reported from their research that the average nurse had moderate compassion satisfaction, 
Astri Eka: The Professional Quality of Life of Nurses who Work in the Intensive Care Unit

burnout, and low secondary traumatic stress. The results interpreted into combination categories and tended to be positive.

This study found that $20.97 \%$ of nurses had a quality of professional life in moderate compassion satisfaction accompanied by burnout and low/moderate stress secondary traumatic. These results are in line with research (Jakimowicz et al., 2017; Kelly \& Lefton, 2017; Kelly \& Todd, 2017) which suggested that nurses are at risk of experiencing fatigue compassion, on the other hand, nurses are not struggling with compassion fatigue, and does not show as a tough worker. Nurses who have a balanced professional quality of life $(24.19 \%)$ in terms of positive and negative aspects, if they cannot maintain or enhance positive aspects and minimize negative aspects, they would be at risk situations. Hegney et al. (2014) revealed that nurses who do not have high compassion satisfaction due to the consequences of burnout or compassion fatigue were influenced by individuals and organizations factors. The individual factor includes the welfare of individual nurses.

The results showed that ER nurses had the lowest average score of compassion satisfaction. According to Sacco and Copel (2017), if hospitals pay attention to welfare and appreciation of nurses, compassion satisfaction would increase and vice versa. RSUD Kota Bandung has the number BLUD (private) nurses larger PNS (the government officer) nurses. Welfare and awards must be given as a form of support system to nurses to help nurses achieve high compassion satisfaction so they have a positive quality of life.

ER nurses had the highest average burnout score. Environmental factors such as stressors and workload factors could make a person experience of burnout (Lumongga, 2016; Stamm, 2010). The number of patients in the ER bigger than the ICU ( 5 bed) and PICU/ NICU ( 4 bed) space keeps patients coming so it is possible to influence the workload of the ER nurse. In addition, unpredictable situations make nurses don't have enough breaks. The number of patients who visit the ER room was 16,551 in 2017. As a result, nurses often did double shifts (morning shifts followed by afternoon shifts), it may be impacted nurses in this room more fatigue compared to other rooms. Fatigue is one sign of burnout that is often felt (Maslach et al., 2001). Stamm (2010) revealed that handling burnout can be done through changes in routine and support systems from people around.

The study found that from the sub-variable secondary traumatic stress, ER nurses have the lowest average score. These results are in line with the Branch study (2015) which showed the average (47.6), this is the lowest ED compared to other rooms. Hooper (2010) in Branch (2015) suggested that emergency room nurses were exposed to trauma or suffering from patients but that exposure occurred briefly because patients were immediately transferred to the nursing room.

This study showed that ICU nurses had the highest average compassion satisfaction score and the lowest average burnout score. This may be influenced by a supportive environment that would enhance compassion satisfaction and prevent nurses from experiencing fatigue compassion (Chen et al., 2017). The supportive environment in the ICU in Bandung City Hospital can be seen from the relationship between the head of the room and staffs. From interviews, nurses said that they felt comfortable working in the ICU.

This study found that the PICU/NICU nurses had the highest average secondary traumatic stress score. These results can be attributed to the characteristics of vulnerable patients requiring help at ages $0-28$ days for NICUs and more than 28 days to 18 years for PICU. In 1995, Figley stated that being a caregiver for children made nurses vulnerable to compassion fatigue. Shen et al. (2015) also stated nurses who work in maternal areas including mother and baby easily exposed to secondary traumatic stress, nurses would do their best to help patients who are in vulnerable condition. The average of the secondary traumatic stress score of ICU nurses lower $(1.04 \%)$ than the average score of PICU/NICU nurses. ICU nurses would be exposed to secondary traumatic stress because the mortality rate in ICU is quite high. Donnelly and Psirides (2015) describes ICU patient mortality internationally, which is $15 \%$ to $24 \%$ this would be affected in nurses' caring behavior. 
Astri Eka: The Professional Quality of Life of Nurses who Work in the Intensive Care Unit

\section{Conclusion}

The percentage of nurses in the ICU unit and ER unit IGD have in Bandung who has a positive Quality of Life City Hospital is low. The overall score of compassion satisfaction is medium to high category. The score of compassion fatigue is low to moderate. ICU nurses have the highest average score in subvariable compassion satisfaction, PICU / NICU nurses have the highest average score in sub-variable secondary traumatic stress, and ER nurses have the highest average score in sub-variable burnout.

Hospitals need to measure Professional Quality of Life nurses regularly. Improving nurse welfare would help nurses to improve compassion satisfaction. The hospital also needs to create a counseling program with a professional psychologist for nurses regularly. Counseling allows nurses to share their problems and obtain help in overcoming their problems.

\section{References}

Ariapooran, S. (2014). Compassion fatigue and burnout in Iranian nurses: The role of perceived social support. Iranian Journal of Nursing and Midwifery Research, 19(3), 279-284.

Austiny, C.L., Saylor, R., \& Finley, P.J. (2017). Moral distress in physicians and nurses: Impact on professional quality of life and turnover. Psychological Trauma: Theory, Research, Practice, and Policy, 9(4), 399406. https://doi.org/10.1037/tra0000201.

Berger, J., Polivka, B., Smoot, E.A., \& Owens, H. (2015). Compassion fatigue in pediatric nurses. Journal of Pediatric Nursing, 30(6), e11-e17. https://doi.org/10.1016/j. pedn.2015.02.005.

Branch, C., \& Klinkenberg, D. (2015). Compassion fatigue among pediatric healthcare providers, 41(June), 161-166.

Braunschneider, H. (2013). Preventing and managing compassion fatigue and burnout in nursing. Essai, 11. Retrieved from http://
dc.cod.edu/essai\%0Ahttp://dc.cod.edu/essai/ vol11/iss 1/11.

Chen, Y.-P., Tsai, J.-M., Lu, M.-H., Lin, L.M., Lu, C.-H., \& Wang, K.-W. (2017). The influence of personality traits and sociodemographic characteristics on paediatric nurses' compassion satisfaction and fatigue. ARPN Journal of Engineering and Applied Sciences, 12(10), 3218-3221. https://doi. org/10.1111/ijlh.12426.

Crewe, C.D., \& Crewe, C. (2016). The watson room: Managing compassion fatigue in clinical nurses on the front line this is to certify that the doctoral study by.

Donnelly, S.M., \& Psirides, A. (2015). Relatives' and staff's experience of patients dying in ICU. Qjm, 108(12), 935-942. https:// doi.org/10.1093/qjmed/hcv059.

Emergency Nurses Association. (2014). ENA topic brief compassion fatigue. Institure for Quality, Safety \& Injury Prevention. Retrieved from https://www.ena.org/docs/ default-source/resource-library/practiceresources/topic-briefs/compassion-fatigue. pdf?sfvrsn=c1bc886d_8.

Figley, C.R. (1995). Compassion fatigue: The stress of caring too much.

Hegney, D.G., Craigie, M., Hemsworth, D., Osseiran-Moisson, R., Aoun, S., Francis, K., \& Drury, V. (2014). Compassion satisfaction, compassion fatigue, anxiety, depression and stress in registered nurses in Australia: Study 1 results. Journal of Nursing Management, 22(4), 506-518. https://doi.org/10.1111/ jonm.12160.

Hooper, C., Craig, J., Janvrin, D.R., Wetsel, M.A., \& Reimels, E. (2010). Compassion satisfaction, burnout, and compassion fatigue among emergency nurses compared with nurses in other selected inpatient specialties. Journal of Emergency Nursing, 36(5), 420-427. https://doi.org/10.1016/j. jen.2009.11.027.

Jakimowicz, S., Perry, L., \& Lewis, J. (2017). Compassion satisfaction and fatigue: A cross- 
Astri Eka: The Professional Quality of Life of Nurses who Work in the Intensive Care Unit

sectional survey of Australian intensive care nurses. Australian Critical Care. https://doi. org/10.1016/j.aucc.2017.10.003.

Kelly, L., \& Lefton, C. (2017). Effect of meaningful recognition on critical care nurses' compassion fatigue. American Journal of Critical Care, 15(2), 130-148.

Kelly, L., \& Todd, M. (2017). Compassion fatigue and the healthy work environment. AACN Advanced Critical Care, 28(4), 351-358. aacnacc2017283.

Lumongga, N. (2016). Depresi: Tinjauan psikologis (2nd Ed.). Jakarta: Kencana. Retrieved from https://books.google.co.id/ books? $\mathrm{id}=\mathrm{p} \_\mathrm{pDDwAAQBAJ} \&$ printse $\mathrm{c}=$ fro ntcover\&dq $=$ Depresi\&hl $=$ en $\&$ sa $=$ X\&ved $=0$ ahUKEwiD7avA5cPZAhUHPo8KHSQECQQ6AEIMDAB $\# \mathrm{v}=$ onepage $\& \mathrm{q}=$ Depresi\&f $=$ false.

Maslach, C., Schaufeli, W.B., \& Leiter, M.P. (2001). Job burnout. Annual Review of Psychology, 52, 397-422. https://doi. org/10.1146/annurev.psych.52.1.397.
Sacco, T.L., Ciurzynski, S.M., Harvey, M.E., \& Ingersoll, G.L. (2015). Compassion satisfaction and compassion fatigue among critical care nurses. Critical Care Nurses, 35(4), 32-43. https://doi.org/10.4037/ $\operatorname{ccn} 2015392$.

Sacco, T.L., \& Copel, L.C. (2017). Compassion satisfaction: A concept analysis in nursing. Nursing Forum, 1-8. https://doi. org/10.1111/nuf.12213.

Schroeder, P. (2011). Current issues in nursing. Journal of Nursing Care Quality, 1. $\quad$ https://doi.org/10.1097/00001786198611000-00012.

Shen, J., Yu, H., Zhang, Y., \& Jiang, A. (2015). Professional quality of life: A crosssectional survey among Chinese Clinical Nurses. Nursing and Health Sciences, 17(4), 507-515. https://doi.org/10.1111/nhs. 12228.

Stamm, B. (2010). The concise ProQOL manual. Pocatello, ID: ProQOL. Org, 78. Retrieved from http://proqol.org/uploads/ ProQOL_Concise_2ndEd_12-2010.pdf. 